\section{Cahiers de Narratologie}

Analyse et théorie narratives

$35 \mid 2019$

Le style comme événement

\title{
Style et événement
}

\author{
Marie-Albane Watine
}

\section{(2) OpenEdition}

Journals

Electronic version

URL: http://journals.openedition.org/narratologie/9195

DOI: 10.4000/narratologie.9195

ISSN: 1765-307X

\section{Publisher}

LIRCES

\section{Electronic reference}

Marie-Albane Watine, «Style et événement », Cahiers de Narratologie [Online], 35 | 2019, Online since 03 September 2019, connection on 25 September 2020. URL : http://journals.openedition.org/ narratologie/9195; DOI : https://doi.org/10.4000/narratologie.9195

This text was automatically generated on 25 September 2020

Article L.111-1 du Code de la propriété intellectuelle. 


\title{
Style et événement
}

\author{
Marie-Albane Watine
}

1 C'est une cause entendue depuis le milieu des années 1970 : l'événement ${ }^{1}$, après sa disgrâce en historiographie, a fait son "retour» dans différentes disciplines des sciences de la culture. La notion a suscité d'importantes publications depuis la fin des années 1990, et montre sa fécondité dans un large champ interdisciplinaire. Pour autant, sa validité n'est pas encore systématiquement évaluée dans le domaine de la stylistique; le présent numéro constitue donc une première exploration de ce croisement, qui, au vu de la richesse des réflexions proposées, s'avère particulièrement fécond. En guise d'introduction, nous nous proposons de retracer le mouvement de la notion au sein des disciplines du sens; nous reviendrons ensuite sur les traits définitionnels originellement proposés dans notre appel à communication, les présupposés et les niveaux de traitement appelés par la proposition de «style comme événement ", en insistant sur les traits consensuels et sur ceux qui ont le plus fait débat; nous mettrons ensuite l'accent sur deux points d'achoppement qui se trouvent au croisement de la plupart des contributions, celui du rapport de l'événement à l'histoire des formes, et celui de l'articulation entre événement et émergence.

2 Mais nous souhaitions aussi faire de ce numéro un jalon pour la poursuite de la réflexion : à la présente synthèse introductive de M.A. Watine, répond l'intervention conclusive d'I. Yocaris, qui, par l'étude des textes et en prenant appui sur les apports des contributions réunies, rouvre le débat en proposant des prolongements possibles aux questions abordées ci-dessous.

\section{Renaissances de l'événement}

3 Au sein des sciences humaines et sociales, l'intérêt pour la notion d'événement n'est pas nouveau, loin s'en faut. La réflexion que nous proposons s'ancre donc dans un ensemble de propositions émanant de champs disciplinaires connexes, propositions qui contribuent à façonner notre vision de l'événement aujourd'hui.

Dans les années 1970, c'est d'une certaine philosophie française qu'est venu le renouveau de la réflexion sur la notion d'événement. Durant plus de trois décennies, les 
plus grands philosophes français ont placé l'événement au cœur de leur pensée, en premier lieu Deleuze, Foucault, Derrida et Lyotard, puis Badiou et Marion. Ricoeur résume cette dignité nouvelle de l'événement, passé de reste de la structure à principe créateur : «l'événement, loin d'être un résidu de ce qui ne se laisse pas systématiser, est initiateur de systèmes, eux-mêmes ouverts à l'aléa » (1992:34). Le travail de Claude Romano, qui dans les années 1990 refonde l'herméneutique sur l'événementialité, "s'inscrit donc dans le prolongement d'[une] longue tradition» (Vinolo 2013: 55); plurielle, cette tradition situe l'événement dans une perspective ontologique chez Badiou (1988) ou plus nettement phénoménologique chez Ricoeur (1992), Romano (1998) ou Marion (2010).

5 Ce corpus philosophique a constitué l'un des points de départ d'un appel à contributions dont nous reprendrons quelques propositions dans la présente introduction; il a surtout initié la réflexion pour plusieurs contributeurs de ce numéro, et se situe au fondement des problématiques de deux contributions, celle de M. Vallespir, qui confronte les apports de Lyotard (1971), Deleuze (1968) et Foucault (1994), et celle de L. Kurts, qui se réfère à l'herméneutique de Romano (1998 et 1999).

Les années 1970 ont vu également la refondation de la notion d'événement en histoire. On sait que l'histoire événementielle héritée du XIX tendait à inscrire une succession de grands faits dans une logique téléologique et causale; et que l'École des Annales a mis à bas «l'idole événementielle » (Simiand 1903) pour lui substituer l'étude du temps long, une histoire sociale des groupes, au regard de laquelle l'événement n'est plus qu'une «agitation de surface» (Braudel 1949: 13). Mais en 1974, le déclin du structuralisme, la diffusion des medias de masse et la rupture de mai 1968 constituent un contexte favorable à la réévaluation de l'événement; dans un article qui a fait date, Pierre Nora proclame le "retour de l'événement ». L'historien y évoque la résonance de Mai 68 dans un monde où les nouvelles communications, et notamment la radio, font vivre à tous l'événement dans son immédiateté: "C'est aux mass media que commençait à revenir le monopole de l'histoire. Il leur appartient désormais. Dans nos sociétés contemporaines, c'est par eux et par eux seuls que l'événement nous frappe, et ne peut pas nous éviter.» (Nora, 1974: 287). Compris comme tel, l'événement, après une éclipse de deux décennies, suscite d'importants travaux en histoire depuis les années 2000 (Farge 2002, Dosse 2010, Rivalan Guégo 2011, Tamm 2015). Comme l'indique l'étude de François Dosse, l'événement qui revient n'est plus celui auquel on a renoncé, et il s'agit moins d'un « retour " que d'une « renaissance »; cet «événement revisité » (Dosse 2010 : 195) est une trace autant qu'une cause et sort ainsi d'un strict rapport à la chronologie. Ce n'est pas la séquence en elle-même qui fait l'événement, mais ses représentations collectives, sa réception continuée qui en filtre et recompose les traces; pour reprendre les mots de Pierre Laborie, " ce qui fait événement n'est pas le seul fait de l'événement ; il n'est pas simplement ce qui advient mais ce qui advient à ce qui est advenu » (Laborie 2011: 168). Il intègre les «reconstructions successives des mémoires collectives de l'événement, indéfiniment revisité » (Laborie 2019 : 27). Dans cette étude des réceptions et des traces, se tisse une « relation essentielle entre langage et événement» (Dosse $2010: 173)$ quile rend compatible avec l'approche discursive, dont relèvent les travaux de Guilhaumou (2006). Ceux-ci sont situés, dans la ligne de la notion d'« événement discursif » développée par Foucault (1969), au croisement de l'histoire et de l'analyse du discours. 
Cette dernière discipline participe logiquement à cette renaissance : le rôle du discours dans la construction sociale des événements est actuellement au centre d'importants travaux sur la circulation des discours, la sémantique et la désignation de l'événement, notamment dans des corpus médiatiques ou archivistiques (Lopez Muñoz et Verine 2012, Calabrese 2013, Londei et al

2013) ; leurs acquis sont intégrés dans le présent numéro par la contribution de Karine Abiven, qui les met en relation avec l'étude serrée de micro-structures stylistiques récurrentes, dans un corpus relatif à un événement historique fondateur.

C'est dans les étudeslittéraires

que la fortune de l'événement s'observe le plus nettement depuis les années 2000 ; l'intérêt s'est notamment porté sur le thème de l'événement dans les corpus littéraires, ou, dans une perspective plus historique ou éditoriale, sur la notion d'événement littéraire. Plusieurs journées d'études se sont tenues sur ces thèmes, à Bruxelles et à Toulouse en 2003 (Alexandre et al.2004), à Rennes en 2004 (Boissetet al.

2016), à Klagenfurt en 2005 (Glaudes et Meter 2008) et à Nice en 2010 (Acquier et Merlo 2012) ; la publication de la thèse de S. Parent en 2004 est aussi le signe de cette vitalité. Deux voies sont tout particulièrement explorées : les modalités de la représentation des événements historiques et naturels dans les textes, et, dans une perspective plus narratologique, le rapport entre la narrativité et la mise en intrigue d'événements

(Baroni 2017).

En comparaison, lesétudes

linguistiques se sont moins saisies de la notion. Alors que Benveniste avait ouvert la voie dès 1963 en considérant notamment que l'énoncé performatif est " événement parce qu'il crée l'événement » (Benveniste, 1963 : 273), l'événement n'a pas fait l'objet

d'une exploration systématique ces dernières décennies, en-dehors des études en analyse du discours mentionnées plus haut. Il est probable que la notion proche d'émergence, récemment exploitée de façon féconde dans les sciences du langage (Badiou-Monferran \& Ducos dirs 2012), a aimanté la recherche. L'un des rares champs de la linguistique à recourir explicitement au terme " événement ", fût-ce dans un sens très restreint, est la neuro-linguistique, avec la mise au jour de certaines modifications remarquables des potentiels électriques produits par le système nerveux en réponse à une stimulation externe. Parmi ces « event-related potentials

", ces potentiels répondant à des événements, la linguistique s'intéresse notamment à la « N400 », qui semble liée à l'anomalie sémantique (Kutas et Hillyard 1980), et à la « P600 », corrélée à l'agrammaticalité (Coulson et al

1998) : il s'agit là d'« événements » situés à une échelle particulièrement micro, et presque toujours inconscients, mais dont l'exploration est riche de promesses.

\section{Stylistique : de quel événement parle-t-on ?}

La recherche stylistique étant fréquemment reliée aux apports de la linguistique, il n'est pas surprenant que l'intérêt potentiel de la notion d'événement pour la pratique et la théorie stylistiques n'ait guère été systématiquement exploré jusqu'ici. Mais le 
moment se prête à une telle réflexion, en raison des présupposés qu'elle comporte sur la notion même de style. Le titre même du numéro, engageant à saisir le «style comme événement ",

suppose de ne pas envisager le style comme un objet d'étude statique, comme un état, mais comme un processus; en accord avec toute une tendance de la stylistique actuelle, dans la lignée de la phénoménologie, de la linguistique de l'énonciation (Rabatel et Petitjean 2007) ou dans celle de la pragmatique (Bonhomme 2005, Jaubert 2007), le style est quelque chose qui survient dans un contexte donné, il « apparait aujourd'hui "en acte", comme l'instrument d'un faire, d'une pratique » (Jenny $2011: 12$ ) : nous renvoyons sur ces points à la conclusion d'Ilias Yocaris, qui développe et illustre par les textes les aspects contextuel et processuel du style.

Cetteinflexion

théorique rend possibles plusieurs déplacements en matière de définition et de qualification des observables stylistiques : pour n'en citer que deux, un premier déplacement, vers la psychologie cognitive, serait l'exploration de l'intersection entre psycho-neuro-linguistique et stylistique (direction notamment explorée dans les travaux de Watine 2017 et 2019, Gautier 2012, Vallespir 2017) ; un deuxième déplacement consiste à intégrer plus systématiquement à la théorie stylistique les dimensions thymiques, anthropologiques ou éthiques du style (voir notamment Rastier 2001, Kurts et Colas-Blaise ici même).

Enfin, le titre du numéro invitait à aborder, outre l'existence éventuelle d'événements de style (ce qui relèverait d'une approche atomiste, ou plus discontinuiste), à la conception même du style comme événement, qui relève d'une approche globale, interactionniste où le style concerne toutes les composantes du discours.

Mais de quel événement parle-t-on ? Comme toutes les notions englobantes et fécondes dans plusieurs champs de la connaissance, l'événement ne se laisse pas définir uniment. L'appel à communication du numéro souhaitait fournir un point de départ à la réflexion, en proposant cinq critères définitoires (largement inspirés du corpus philosophique) et en appelant à les discuter. Nous reprenons ici les termes de ces propositions :

«- Imprévisibilité : l'événement s'annonce avec une absolue nouveauté, dans la mesure où il semble inexplicable à partir des possibles qui lui préexistent.

$$
\text { où il semble inexplicable à partir des possibles qui lui préexistent. }
$$

- Sur-signification : l'événement, bien que déjouant toute attente, est perçu comme particulièrement signifiant.

\section{- Rupture : l'événement représente un tournant radical dans l'expérience : «} après le surgissement de l'événement, ce ne sera plus jamais comme avant » (Romano

1998 : 62).

- Intensité : l'événement suppose une intensité dans l'ordre émotionnel, esthétique, intellectuel, qui dépasse le ressenti d'une simple surprise.

- Adresse : l'événement est ce qui arrive à quelqu'un, sollicite son attention, ses ressources cognitives; au-delà, il l'institue comme un sujet particulier, celui à qui arrive l'événement, et qui y répond - voire même celui qui advient par l'événement. »

(Watine et Yocaris 2017) 
Certains de ces éléments, comme l'adresse ou le caractère particulièrement signifiant, semblent repris sans difficulté par les contributions ici réunies, au prix éventuellement de quelques déplacements. En revanche, d'autres sont régulièrement discutés, amendés ou remis en question : c'est en particulier le cas pour le critère de la rupture, et dans une moindre mesure celui de l'intensité : nous y reviendrons.

Une réticence du même ordre s'est manifestée sur le plan des domaines où l'appréhension du style comme événement s'est trouvée le plus débattue. En effet, l'événement, en matière de style, peut survenir à différents niveaux. Nous en proposions quatre dans l'appel à contribution, étroitement reliés entre eux : le niveau textuel

des faits ou des interactions concernés en termes formels (génériques, énonciatifs, grammaticaux...) ; celui de la réception

, qui dans certaines configurations spécifiques peut s'appréhender en termes d'événement attentionnel, perceptif, cognitif ; celui, historique

, de la pertinence de la notion d'événement en matière d'histoire des formes et de changement stylistique ; celui, méta-théorique, de l'événementialité de la théorisation stylistique elle-même.

Le niveau textuel - préalable méthodologique de toute stylistique - est diversement exploité par toutes les contributions ; on évalue ainsi l'événementialité potentielle d'un large éventail de faits, parmi lesquels les figures de sens (M. Bonhomme et $\mathrm{M}$. Vallespir), les morphèmes grammaticaux (N. Laurent), les marques graphiques (M. Colas-Blaise), l'ancrage énonciatif et les représentations sémantiques pathiques ( $M$. Monte), la mise en discours et de la désignation de l'événement historique (K. Abiven). Le caractère adressé de l'événement dans la plupart de ses définitions (« l'événement

arrive à quelqu'un

») impliquait tout particulièrement un questionnement sur l'interaction du texte avec son lecteur : la question des modalités de la réception d'un événement de style, présente à l'arrière-plan de toutes les contributions, est notamment caractérisée en termes neuro-cognitifs chez M. Vallespir, tandis que S. Vaudrey-Luigi explore le rôle de la mise en scène médiatique dans ce qui se donne comme événement de style, et que $\mathrm{M}$.

Bonhomme examine l'activité herméneutique en jeu dans le repérage de la figure. Le versant symétrique de la production est documenté dans l'étude de M. Monte, qui montre dans quelle mesure et à quelle condition la récriture d'un texte peut constituer un événement, en mettant tout particulièrement en évidence la dimension processuelle du style. Le niveau métathéorique est peu exploré dans ce numéro - bien que Christelle Reggiani évoque l'événementialité de la reconnaissance théorique et terminologique des phénomènes stylistiques. 
C'est indiscutablement le niveau historique qui suscite le plus de polémique. Les contributions de C. Reggiani, C. Badiou-Monferran et S. Vaudrey-Luigi, notamment, montrent toutes les réticences que l'on peut opposer au concept d'événement de style dès lors que le style est envisagé dans son historicité. La possibilité (ou non) d'entendre le style comme événement au niveau historique rejoint la discussion autour de l'un des traits définitionnels proposé pour l'événement, celui de la rupture : elles impliquent la temporalité de l'événement, et son caractère de seuil disruptif. Ainsi, l'une des questions les plus débattues du numéro peut se résumer ainsi : l'événement fait-il date? et, corrélativement, la rupture est-elle pertinente pour aborder l'évolution des

\section{Style, événement et historicité}

Il ne s'agit pas ici de trancher définitivement une question délicate, mais de souligner les grandes lignes d'un débat qui appelle sans nul doute à être poursuivi. L'une des pistes ouvertes dans l'appel à contribution était celle du changement stylistique : "Quand peut-on dire qu'une œuvre, qu'un style fait événement dans l'histoire littéraire ? Peut-on articuler théoriquement l'imprévisibilité de l'événement, son caractère hors-cause, hors-série, avec l'historicisation des « patrons » stylistiques (Philippe et Piat 2009) ? ». Comment situer l'événement par rapport à l'évolution des formes ? L'événement est-il un certain point de vue sur le changement stylistique ? Ou ne désigne-t-il qu'un type de changement ? Est-il même une notion pertinente pour penser ce changement?

Or, plusieurs contributions tendent à répondre par la négative à cette dernière question. À cet égard, C. Reggiani et S. Vaudrey-Luigi, même si elles partent de postulats différents, se rejoignent sur ce point : la notion d'événement, parce qu'elle tendrait selon elles à maximaliser les effets de rupture, ne rendrait pas compte de

l'histoire des formes, qui est graduelle, complexe et peu singulière. La notion d'événement stylistique survaloriserait indument la nouveauté, les sauts et les seuils, et relèverait donc d'un préjugé avant-gardiste que l'étude serrée des formes dans leur développement historique comme la leçon de l'histoire de l'art tendraient à invalider. Dans une telle perspective, l'événement stylistique est fortement corrélé à la notion de style d'auteur (ce qui fait événement, aux yeux du public, c'est une forme inventée par tel auteur à tel moment) ; or les travaux de G. Philippe, auxquels se réfèrent les contributrices, ont largement contribué à la remise en cause des excès de la stylistique auteuriste (Philippe 2017, Philippe et Piat 2018), et mettent en garde contre la notion d'événement stylistique, qui tend à surjouer le mythe du grand auteur, l'originalité et la singularité, quand un grand nombre de changements relèvent en réalité de dynamiques plus subtiles et plus collectives. Dans ce débat, on trouve une réminiscence du procès de l'histoire événementielle : il n'est pas impossible de faire le parallèle entre

la remise en cause du rôle du grand auteur et la critique par l'École de Annales du " péché événementialiste » (Braudel 1958), qui tend à attribuer aux grands hommes et aux grandes batailles la responsabilité du mouvement de l'Histoire, quand celui-ci relève des structures sous-jacentes, des groupes sociaux et de la longue durée. 
L'objection, particulièrement fondée, doit être entendue. Comme toute critique de valeur, elle appelle deux types de réponses ; la première consiste à envisager, plus que des réfutations, des nuances, touchant à l'extension de l'objection - en d'autres termes, existe-t-il des conditions, des époques ou des niveaux auxquels la notion d'événement resterait pertinente, s'agissant du changement stylistique ? La deuxième consiste à revenir sur les caractéristiques définitoires de l'événement, pour réévaluer ou reformuler certains de ses critères.

Pour ce qui est de la pertinence laissée à l'événement dans le cadre d'une histoire des formes, deux directions sont esquissées dans ce numéro. D'une part, si la pertinence de

l'événement (à tout le moins s'il est envisagé sous un angle disruptif) est sujette à caution pour les périodes d'ancien régime, il n'en va pas de même pour les périodes où le champ littéraire se structure autour d'avant-gardes : la validité de l'événement serait donc affaire de moment. C'est ici la position défendue par C. Badiou-Monferran. D'autre part (mais ces deux orientations ne sont pas compatibles), il est également possible, en se déplaçant d'une perspective ontologique (considérant les seuls observables textuels)

vers une perspective plus phénoménologique (prenant en compte la perception des phénomènes par les instances lectrices, critiques, médiatiques) de considérer que, si le changement des formes est trop graduel pour être pensé en termes d'événement, son mode de perception comporte parfois la soudaineté et l'imprévisibilité de l'événement :

la reconnaissance d'une forme par un public, en ce sens, fait événement - quand bien même l'apparition graduelle de la forme, elle, n'en était pas un. C'est vers cette

conclusion que tendent par exemple M. Monte et $\mathrm{C}$. Reggiani. Appliqué à l'étude du corpus durassien menée par S. Vaudrey-Luigi, cet aménagement pourrait être formulé ainsi : les formes mobilisées par

L'Amant

ne sont ni propres à Duras, ni irruptives dans sa prose, mais (en raison notamment d'un certain dispositif de communication) leur reconnaissance est bien de l'ordre de l'événement. C'est, d'une certaine façon, replacer au centre de la réflexion une dimension majeure dans la définition de l'événement : son adresse - nous y reviendrons. C'est aussi rejoindre la réflexion des philosophes pragmatistes américains comme George Herbert Mead ou John Dewey dans les années 1920, qui postulaient une distinction entre le changement ou événement existentiel, qui se pense sous les catégories du devenir et de la transition, et l'événement objet, qui fait « saillie » pour un observateur et relève d'une perception et d'un jugement (Quéré 2013).

L'événement qui, depuis 30 ans, a été remis à l'honneur en histoire, est devenu l'un des prismes majeurs de la perception du changement dans cette discipline ; il semble donc qu'il ne devrait pas être entièrement déplacé, s'agissant de stylistique historique. C'est sans doute que les critiques formulées à son encontre mettent au jour les limites des critères définitoires proposés initialement, et qu'il s'agit à présent de faire retour sur certains d'entre eux pour les réévaluer. Nous commencerons par un trait que nous n'avions pas mentionné, mais qui est souvent attaché à la notion d'événement : sa singularité. Si cette singularité est comprise comme l'équivalent de "l'individualité ", alors elle désigne ce qui émane d'un individu (auteur) donné, et la notion d'événement de style reconduit alors les apories d'une stylistique réduite à l'étude des idiosyncrasies

- mais il faut affirmer ici que rien n'oblige à assimiler singularité et individu, et

l'événement ne comporte pas obligatoirement le risque de fixation auteuriste qu'on 
Il importe, enfin et surtout, de revenir sur le trait le plus controversé de l'événement : le caractère de "rupture" que nous soumettions à la réflexion. Si M. Bonhomme montre que la rupture est productive sous l'angle des événements de style que sont les figures de sens, plusieurs contributions (notamment L. Kurts, M. Colas-Blaise, M. Vallespir, M. Monte) s'attachent à discuter ce trait. A côté, ou à la place de l'événement disruptif, imprévu, opérant une césure distinctive dans le temps, elles imaginent, aux différents niveaux où elles se placent, un événement plus continuiste, tensif, graduel, fruit de modifications invisibles et constantes: autant un événement qu'un « avènement continu » (Julien 2009: 156), qui semble éclater quand son intensité ou sa fréquence sont maximales, ou qu'il devient perceptible en raison d'une convergence de conditions contextuelles.

Peut-on penser un événement peu ou non-disruptif ? Notons que, si la philosophie des trois dernières décennies insiste sur cette disruptivité, convoquée aussi bien chez

Badiou que chez Romano ou Derrida, elle est beaucoup moins impliquée par la définition courante du mot. Pour le TLF, l'événement est (de façon peut-être un peu minimaliste) « tout fait qui s'insère dans la durée », un « fait d'une importance notable pour un individu ou une communauté humaine », ou un « fait qui attire l'attention par son caractère exceptionnel »: la perception et l'adresse priment sur la fracture.

La possibilité de penser un événement plus continuiste et moins intense semble en tout état de cause convergente avec la redéfinition contemporaine de l'événement en

histoire. Les historiens envisagent actuellement des événements plus continus, plus ténus, moins extraordinaires ; dans le sillage de Paul Veyne (1978), Arlette Farge promeut l'étude des « événements qui n'en sont pas, ou plutôt des séries d'événements fabriqués par les "faibles intensités" des mouvements humains et sociaux »: «il est des événements faibles et fragiles, sans grande ampleur, qui sont en quelque sorte les "un peu" de l'histoire » (Farge 2002). Cette démarche tend à conférer aux non-événements le statut de l'événement, façon de réduire la tension connue, en philosophie de l'histoire, entre la succession des événements et le caractère continuiste de l'histoire.

La redéfinition de l'événement historique offre également des pistes pour prolonger la réflexion touchant aux rapports entre chronologie et changement stylistique. L'histoire des formes est faite d'anticipations et de survivances, qui s'accomodent mal de la notion d'événement, si celui-ci découpe uniment un avant et un après. Mais l'histoire contemporaine, en insistant sur la construction discursive de l'événement en temps réel, qui en fait un objet « fondamentalement tributaire d'une mise en récit composée a posteriori », met en lumière le «télescopage des temporalités » (Dosse 2010:63) qu'il produit. À la fois fruit d'une maturation en amont et signe d'une attente de ce qu'il préfigure, toujours recomposé dans la suite de ses saisies, "construction permanente qui se développe dans la durée selon des temporalités plus ou moins longues et fragmentée » (Capdevilla $2004:$ 85), il justifie un usage raisonné de l'anachronisme en histoire (Dosse 2005) - et paraît dès lors un peu plus propre à saisir la temporalité complexe de l'histoire des styles.

Mais, si l'événement peut voir ses caractères de rupture ou de forte intensité remis en cause, s'il peut être rapproché dans certains contextes des notions de flux et d'évolution, la question se pose de savoir s'il est l'autre nom d'une notion proche, celle d'émergence. 


\section{Événement et émergence}

L'intérêt pour l'émergence dans le champ des sciences de la culture coïncide chronologiquement avec le « retour de l'événement ». Comme l'événement, l'émergence pose de façon centrale la question de la nouveauté et du changement ; davantage que lui, elle a suscité depuis la fin du siècle dernier un nombre important de publications en linguistique, notamment dans le domaine de l'acquisition (MacWhinney 1999, Kail et Fayol 2000), dans l'ensemble des sciences du langage (Badiou-Monferran et Ducos 2012), et récemment en stylistique, notamment chez I. Yocaris (2016a et b). Nous laissons ce dernier, dans sa contribution conclusive, développer ce point plus avant, en s'appuyant sur des analyses textuelles de détail : nous nous contenterons de proposer ici une synthèse des diverses propositions et une reformulation des termes du débat.

Émergence et événement possèdent assurément des caractéristiques communes ; leur articulation, nécessaire, prend dans ce numéro des formes allant de l'assimilation à la contrariété, en passant par l'inclusion. Plusieurs contributions en font des quasisynonymes - ou, en adéquation avec le sémantisme de leurs suffixes respectifs (entet -ence

), l'une est donnée comme le mode d'apparaître de l'autre : ainsi, en général ou sous certains régimes, un « événement émerge " - ou, à l'inverse, suivant l'articulation d'Edgar Morin, les émergences ont « vertu d'événements » (1977 : 108). Pour C. Reggiani, au contraire, les deux termes, exclusifs l'un de l'autre, désignent des réalités d'ordre différent : l'émergence, par la gradualité qu'elle implique, est seule apte à caractériser le changement stylistique, tandis que l'événement peut désigner la reconnaissance théorique de la nouveauté. Chez C. Badiou-Monferran, l'émergence englobe l'événementialité propre à la post-modernité, et la variation propre à l'Early Modern.

Synonymie, antonymie ou hypéronymie ? Il est sans doute trop tôt pour trancher la question, qui relève autant de l'examen des observables stylistiques que d'un travail définitionnel - au cœur duquel figure sans doute la question de la gradualité (ou non) de l'événement. Nous nous contenterons ici de rappeler une distinction épistémologique touchant à l'histoire de ces deux notions, et d'insister sur un trait qui nous paraît faire la différence : l'adresse.

D'un point de vue archéologique, événement et émergence n'ont pas le même contexte

d'apparition. Alors que l'événement, nous l'avons vu, relève initialement d'un paradigme historique puis philosophique, qui se diffuse au sein des sciences de la culture vers l'anthropologie, la sociologie, l'analyse de discours et les études littéraires, la notion d'émergence apparaît dans un tout autre contexte. On fait remonter son apparition à la reformulation par Lewes (1875) de la Logique 
de Mill (1843), où l'émergence peut s'appréhender par l'aphorisme connu " Le tout est plus que la somme des parties » : c'est la relation qui se noue entre les propriétés d'un objet et celle de ses parties, quand les premières ne sont pas dérivables des secondes.

Lewes donne l'exemple, souvent repris, de la transparence de l'eau, impossible à prédire à partir des propriétés de l'oxygène et de l'hydrogène. En tant qu'apparition de propriétés complexes et nouvelles dans le champ du vivant, l'émergence se diffuse ensuite dans l'étude de l'évolution biologique, et notamment dans la reformulation des thèses darwiniennes par Morgan (1923) : l'évolution n'est pas un processus continu, elle comporte des points de rupture où apparaissent des propriétés radicalement nouvelles, émergentes, comme la vie ou la conscience. Mais l'émergentisme connaît, dès la fin des années 1920, une éclipse liée à la domination du physicalisme réductionnisme. Les années 1970 sont propices à la « ré-emergence de l'émergence » (Cunningham 2001), notamment en physique, en biologie, dans le domaine de l'intelligence artificielle

; la notion se diffuse plus marginalement en sciences sociales (Sawyer 2005), ou en économie avec la notion de " marché émergent ", ou encore, on l'a vu, en linguistique.

C'est donc originellement au sein des sciences de la nature, ou de la philosophie des sciences naturelles, que naît la notion d'émergence, et aujourd'hui encore c'est surtout dans ces domaines qu'elle est redéfinie, aménagée ou contestée. Le corollaire de cet ancrage, c'est que les propriétés émergentes relèvent des propriétés des systèmes où elles apparaissent, bien plus que de l'appréhension de ces propriétés par les sujets qui les reçoivent - notamment dans la version « ontologique » (ou « forte ») de l'émergence, qui place celle-ci dans le monde naturel, tandis que la version " épistémologique » (ou « faible ») attribue les propriétés émergentes à nos modalités de représentation du monde. Mais, dans l'une ou l'autre version, les définitions de l'émergence reposent sur une description (souvent mathématique, ou logique) de propriétés, qui comprennent souvent l'accroissement de complexité, l'hétérogénéité des effets et des causes, et la rétroaction des effets sur les causes ; la question de la réception de l'émergence, de ses effets sur les sujets, ne fait pas partie de ses traits. 
Cette question a pu se poser à propos de l'événement - dont il existe, notamment chez Badiou, une version ontologique, et une version phénoménologique. Pour reprendre la synthèse de $\mathrm{S}$. Vinolo, « ou bien nous acceptons de penser l'événement sur un horizon ontologique, et nous pouvons alors dire qu'à proprement parler, il y a, dans l'être, des faits non-événementiels et des événements. [...] Ou bien au contraire, nous acceptons, comme le fait la majorité des penseurs de l'événement, que le sujet [...] est impliqué de quelque façon dans la reconnaissance des événements, et que l'événementialité relève ce faisant plus d'une phénoménologie que d'une ontologie » (2013:52). Les contributeurs du numéro se positionnent assurément sur l'option phénoménologique, et reprennent à leur compte cette dimension adressée de l'événement, sa saillance perceptive, son traitement cognitif, ses implications herméneutiques, émotives et esthétiques. De par son histoire et son ancrage disciplinaire, la notion d'événement intègre plus de façon plus centrale la subjectivité ou l'intersubjectivité impliquées par

la stylisation et le processus de réception esthétique, quand la définition logique de l'émergence rend peut-être plus difficile la prise en compte des dimensions perceptives, émotives et esthétiques du style - sauf à rattacher celles-ci aux critères de complexité, d'inscription dans la durée ou de rétroaction. Le débat reste ouvert : nous renvoyons, pour des prolongements illustrés par les textes, à la contribution conclusive d'I. Yocaris, et naturellement à l'ensemble des contributions.

\section{Résumé des contributions}

Karine Abiven entend explorer les rapports qui se nouent entre événement stylistique et événement historique. À ce titre, elle se penche sur le traitement discursif d'un épisode historique lié à la Fronde : il s'agit de l'érection de barricades à Paris en août 1648, événement qui a suscité une abondante production discursive. Son objectif consiste à mettre au jour certains faits de langue qui se constituent discursivement en événements de style, dès lors qu'ils apparaissent comme des moyens expressifs récurrents dans l'ensemble hétérogène d'écrits qu'elle a choisi de retenir. Elle s'attache donc à montrer que des points communs formels traversent les différentes pratiques socio-discursives, l'événement se constituant aussi par une langue partagée. Dans cette optique, elle analyse successivement les différentes dénominations des événements survenus en août 1648, les figures du renversement liées aux rebondissements successifs narrés dans les textes de son corpus, l'emploi des discours rapportés, le recours à des tournures passives et l'utilisation des indéfinis.

En prenant pour observatoire l'entrée formelle des et «de relance», constamment sollicités par les écrivains français de Rabelais à Duras, Claire Badiou-Monferran se propose d'éprouver la validité (descriptive et explicative) de la proposition d'« événement de style» en diachronie. Comme elle le montre, cette modélisation, élaborée par Yocaris (2016a) et Watine \& Yocaris (2019), rencontre certaines difficultés à décrire les corpus de l'ancien régime littéraire (XVIe-XVIIIe siècles), soumis selon elle à une logique moins "événementielle» que "variationniste». Dans la perspective d'une histoire du changement stylistique tout à la fois discrète et continuée, elle interroge dès lors la capacité du modèle de l'« émergence » (Badiou-Monferran \& Ducos 2012), à prendre en charge l'entier de cette histoire, et à penser l' « événementialisation » de la langue littéraire des Belles-Lettres. 
Pour sa part, Marc Bonhomme questionne l'articulation entre le style littéraire et le concept d' "événement" à travers le cas d'une des figures du discours les plus représentatives : la métonymie. Celle-ci est redéfinie comme un "événement de style ", en ce qu'elle constitue une intrusion à la fois déstabilisante et hautement signifiante à la surface des textes. Dans un premier temps, il décrit la production de métonymies comme un événement de style disruptif, en raison des ruptures communicationnelles, des saillances textuelles et des tensions sémantiques que leur émergence suscite au sein des occurrences littéraires. Dans un second temps, il s'intéresse à la réception des énoncés métonymiques, envisagée comme un événement de style herméneutique du fait du caractère aléatoire de leur identification et de leur interprétation selon les particularismes des différents contextes. Par ailleurs, il analyse l'activité de stylisation des lecteurs, qui consiste à donner du sens et une pertinence esthétique à la singularité des métonymies identifiées. Au total, une telle approche événementielle d'une figure comme la métonymie met en évidence son irréductibilité discursive en fonction des pactes de lecture instaurés par chaque texte littéraire.

Dans sa contribution, Marion Colas-Blaise entreprend une réflexion théorique approfondie sur la notion d' " événement de style ", en mettant la notion d'écart, revisitée, en relation avec une double logique (concessive et implicative), qui sous-tend respectivement l'impertinence et l'hyperpertinence. Comme elle le montre, le mérite de la notion de saillance réside dans la mise en avant d'une appréhension sensible de l'événement de sens, dont l'« énergétique du mot » italiqué selon Julien Gracq offre un exemple saisissant. Conjointement, il est possible de penser le style comme événement, en mettant en avant trois composantes du style: la pratique textualisante, la praxis énonciative (générique par exemple) et l'expérience sensible. Le style constitue ainsi in fine un événement dans l'exacte mesure où il est porté par une forme de vie.

L'article de Lia Kurts se situe également sur le terrain de la sémiotique, et notamment la sémiotique des cultures de $\mathrm{F}$. Rastier. Elle entend articuler théoriquement le style, vu comme le résultat d'un processus d'individuation, avec le concept classique de manière, tel qu'il a été repris et réévalué dans l'approche poétique de G. Dessons. Articulant la définition philosophique de l'événement chez Claude Romano avec la valeur événementielle de la lecture, elle examine ensuite la dimension événementielle que peut revêtir la notion de manière - qui a pu dans une certaine mesure répondre aux excès techniciste de la notion de style. Elle intègre enfin la manière dans le concept de "style sémiotique ", qui prend en considération le processus d'individuation général dont relève le style. Le projet global d'une herméneutique modale invite à relier les œuvres, conçues comme événements artistiques, à l'aventure de leur réception.

Nicolas Laurent se focalise sur la dimension émergentiste des faits de style, en mettant en évidence leur ancrage référentiel : ils contribuent à une mise en tension de la langue et $\mathrm{du}$ "monde», formant ce qu'on peut appeler un événement de représentation. S'inspirant notamment des travaux d'Edgar Morin sur la complexité, il montre que l'événementialité du style littéraire repose sur une interrelation complexe entre les différentes composantes d'un texte donné et l'œuvre dont elles font partie intégrante : le style apparaît dès lors comme une qualité émergente globale, mais aussi comme un mode d'organisation significatif impliquant des paliers textuels différents. Fort de cette définition, N. Laurent étudie ensuite les procédés de stylisation liés à l'emploi des morphèmes et des traits grammaticaux, dont le sens peut engendrer des effets de figuration et d'implication prédicative : comme il le souligne, ceci apparaît clairement 
quand on se penche par exemple sur l'emploi de la préposition en, la construction phrastique et les particularités de la flexion verbale dans les textes pascaliens.

Prenant le contre-pied d'une telle approche, Christelle Reggiani défend l'idée que les faits de style ne sont pas forcément des événements en soi, en dépit de leur dimension émergentiste : partant de cette hypothèse de travail, elle propose une définition globale du style axée sur le concept goodmanien d'exemplification. À ses yeux, le style littéraire se présente comme un ensemble de propriétés formelles découlant d'une dialectique entre des choix scripturaux individuels (qu'ils soient ou non concertés) et des imaginaires langagiers et esthétiques collectifs stabilisés en patrons stylistiques: comme le montre Gilles Philippe, ces imaginaires orientent de façon constitutive la stratégie discursive d'un auteur donné, fût-ce quand celui-ci tente progressivement de s'en affranchir. Les faits de style ne sont pas toujours des événements ponctuels et distinctement localisables, puisqu'ils s'inscrivent dans une temporalité linguistique et esthétique complexe: en effet, l'interaction dialogique entre un patron stylistique collectif et une écriture singulière qui tend à le dépasser peut générer dissonances décalages chronologiques.

Dans un autre ordre d'idées, Michèle Monte aborde les rapports entre style et événementialité sous un angle génétique, en examinant les modifications apportées à trois poèmes de Pierre Dhainaut issus de l'anthologie Dans la lumière inachevée. La réécriture de ces poèmes, déjà publiés auparavant dans Un livre d'air et de mémoire, est envisagée comme un événement en ce sens qu'elle affecte le texte d'une série de changements dont il importe d'analyser l'impact, et, si possible, de restituer la cohérence. Cette réflexion est prolongée par un questionnement sur la façon dont l'événement qui a présidé à l'écriture du poème est ressaisi des années plus tard et sur la part que prennent les caractéristiques stylistiques propres au texte initial dans l'opération de relecture et de réécriture. Sont ainsi abordés des faits de style liés au rythme, à la configuration isotopique des poèmes analysés, à leur ancrage énonciatif, leur orientation argumentative.

Mathilde Vallespir, quant à elle, met en œuvre une approche transdisciplinaire des rapports entre style et événement. Dans son étude, elle s'attache à montrer que les événements stylistiques ont partie liée avec le concept de "multistabilité », en convoquant les travaux de certains philosophes postmodernes et l'approche neurophénoménologique de Francisco Varela pour les articuler ensemble. Après avoir circonscrit les caractéristiques de l'événement selon Deleuze, Foucault et Lyotard, elle met en évidence la parenté de leurs définitions de l'événement avec la notion varélienne d'énaction. Ce rapprochement lui permet d'envisager l'événement comme "plateau multistable». Pour illustrer son propos, elle convoque la figure choisie par Lyotard pour exemplifier sa définition de l'événement figural, «je te musique », et de l'analyser dans une nouvelle perspective, à l'aune de l'approche énactive de F. Varela. Elle souligne ainsi que l'énoncé en question donne lieu à un agencement en "plateau multistable ", lequel, par ses propres prérogatives et dans ce nouveau cadre, est à son tour susceptible de faire événement au sein du texte.

Tout comme C. Reggiani, Sandrine Vaudrey-Luigi s'inspire des travaux de Gilles Philippe sur la phrase littéraire, pour discuter et remettre en cause la notion d'événement appliquée à des évolutions stylistiques. Son exposé s'appuie sur une étude du style de Marguerite Duras. Comme elle le montre, l'évolution de l'écriture durassienne sur le plan diachronique (entre les deux points de repère chronologiques 
que sont Un barrage contre le Pacifique en amont et L'Amant en aval) a partie liée avec deux tendances: d'une part, la mise en œuvre d'une série d'expérimentations langagières portant notamment sur la construction phrastique (qui tend à s'éloigner de plus en plus des normes grammaticales habituelles); d'autre part, des réminiscences de la «belle langue ", autrement dit du français littéraire académique dont des traces subsistent encore dans les récits durassiens... Or, le « dosage » entre ces tendances varie constamment en fonction de l'évolution des patrons régissant l'écriture littéraire lors de la période 1950-1990 et du positionnement fluctuant de Duras par rapport à ces patrons - ce qui tend à invalider l'absolue nouveauté stylistique de L'Amant, telle qu'elle a été mise en avant par l'auteur elle-même.

\section{BIBLIOGRAPHY}

Acquier, Marie-Laure \& Merlo, Philippe dirs (2012) : La Relation de la littérature à l'événement, XIXeXXIe siècles, Paris, l'Harmattan.

Alexandre, Didier, Frédéric, Madeleine, Parent, Sabrina et Touret, Michèle dirs (2004) : Que se passe-t-il ? Événements, sciences humaines et littérature, Rennes, Presses universitaires de Rennes, coll. « Interférences ».

Badiou, Alain (1988) : L'Être et l'événement, Paris, Le Seuil.

Badiou-Monferran, Claire et Ducos, Joëlle dirs (2012) : L'Émergence : un concept opératoire pour les sciences du langage?, L'Information grammaticale, 134.

Baroni, Raphaël (2017) : Les Rouages de l'intrigue. Les outils de la narratologie postclassique pour l'analyse des textes littéraires, Genève, Slatkine.

Benveniste, Émile (1966) : Problèmes de linguistique générale, Paris, Gallimard.

Boisset, Emmanuel et Corno, Philippe (2016) : Que m'arrive-t-il ? Littérature et événement, Presses Universitaires de Rennes.

Bonhomme, Marc (2005) : Pragmatique des figures du discours, Paris, Champion, coll. « Bibliothèque de grammaire et de linguistique ».

- (2008) : " Pour une intégration modulaire de la stylistique dans une linguistique de l'écrit », in Jacques Durand et al. dirs, Congrès Mondial de Linguistique Française, Paris, Institut de Linguistique Française, p. 1491-1495, http://dx.doi.org/10.1051/cmlf08327.

Braudel, Fernand (1949) : La Méditerranée et le Monde méditerranéen à l'époque de Philippe II, Paris, Colin.

- (1958) : «La longue durée », Annales ESC, n4, pp.725-753.

Calabrese, Laura (2013) : L'Événement en discours. Presse et mémoire sociale. Académia-L'Harmattan, coll. « Science du langage. Carrefours et points de vue », Louvain-la-Neuve.

Capdevila, Luc (2004) : «Les temporalités de l'événement en histoire », in Alexandre, Didier, Frédéric, Madeleine, Parent, Sabrina \& Touret, Michèle dirs, Que se passe-t-il ? Événements, sciences humaines et littérature, Rennes, Presses universitaires de Rennes, coll. «Interférences », p. 79-89. 
Coulson, Seanna, King, Jonathan et Kutas, Marta (1998) : « Expect the unexpected : event-related brain response to morphosyntactic violations ", Language and Cognitive Processes, $n^{\circ} 13$ (1), p. 21-58. Deleuze, Gilles (1968) : Logique du sens, Paris, Minuit.

Dosse, François (2005) : « De l'usage raisonné de l'anachronisme », Espace Temps, 87-88, p. 156-171.

- (2010) : Renaissance de l'événement. Un défi pour l'historien : entre sphinx et phénix, Paris, PUF.

Fagot-Largeault, Anne (2002) : «L'émergence », in Andler, D., Fagot-Largeault, A. et Saint-Sernin B., Philosophie des sciences II, Paris, Gallimard, coll. « Folio », p. 939-1048.

Farge, Arlette (2002) : « Penser et définir l'événement en histoire », Terrain [En ligne], 38, mars 2002, https://journals.openedition.org/terrain/1929.

Foucault, Michel (1969) : L’Archéologie du savoir, Paris, Gallimard.

— (1994) : Dits et écrits 1954-1988, III, 1976-1979, Paris, Gallimard.

Gautier Antoine (2012) : " "La Pause et l'effet" : hyperbate et segmentation graphique », in AnneMarie Paillet et Claire Stolz dir., L'Hyperbate. Aux frontières de la phrase, Paris, PUPS, p. 103-116.

Glaudes, Pierre et Meter, Helmut (2008) : Le sens de l'événement dans la littérature française des XIX et XXe siècles, Bern, Peter Lang.

Guilhaumou, Jacques (2006) : Discours et événement. L'histoire langagière des concepts, Besançon, Presses Universitaires de Franche-Comté.

Jaubert, Anna (2007) : « La diagonale du style », in Rabatel \& Petitjean dirs, Pratiques, 135-136, Questions de style, p. 47-62.

Jenny, Laurent (2011) : « Introduction », in Laurent Jenny dir, Le Style en acte. Vers une pragmatique du style, Genève, MetisPresses, p. 11-15.

Kail, Michèle et Fayol, Michel dirs (2000), L'acquisition du langage : le langage en émergence, de la naissance à trois ans, Paris, PUF, 2000.

Kutas, Marta et Hillyard, Stephen A. (1980) : « Reading senseless sentences : Brain potentials reflect semantic incongruity », Science, $\mathrm{n}^{\circ} 207$, p. 203-208.

Laborie, Pierre (2011) : «L'événement, c'est ce qui advient à ce qui est advenu... », entretien avec Pascale Goetschel et Christophe Granger, Sociétés et Représentations, n³2, 2011, p. 167-181.

- (2019) Penser l'événement. 1940-1945, Paris, Folio Histoire.

Lewes, George Henry (1875) : Problems of Life and Mind (Vol. II), London, Trübner \& co.

Londei, Danielle, Moirand, Sophie, Reboul-Touré, Sandrine \& Reggiani, Licia dirs (2013) : Dire l'événement : langage mémoire société, Paris, Presses Sorbonne nouvelle, 2013.

Lyotard, Jean-François (1985 [1971]) : Discours, figure, Paris, Klincksieck.

MacWhinney, Brian dir. (1999) : The Emergence of Language, New Jersey, Lawrence Erlbaum.

Marion, Jean-Luc (2010) : Certitudes négatives, Paris, Grasset.

Mill, John Stuart (1843) : A System of Logic, Ratiocinative and Inductive, Being a Connected View of the Principles of Evidence and the Methods of Scientific Investigation, London, John W. Parker.

Morgan, Conwy Lloyd (1923) : Emergent Evolution, London, Williams \& Norgate. 
Nora, Pierre (1974) : « Le retour de l'événement », in J. Le Goff et P. Nora dirs, Faire de l'histoire, t.1, Paris, Gallimard, 1974, p. 285-308 [version remaniée de «L'événement monstre », Communications $\left.n^{\circ} 18,1972\right]$.

Parent, Sabrina (2011) : Poétiques de l'événement : Claude Simon, Jean Rouaud, Eugène Savitzkaya, Jean Follain, Jacques Réda, Paris, Garnier.

Philippe, Gilles (2017) : « Parce qu'un nouvel auteur survient? Quelques réflexions sur le changement stylistique », Poétique n¹81, p. 5-17.

Philippe, Gilles et Piat, Julien dirs (2009) : La Langue littéraire. Une histoire de la prose en France de Gustave Flaubert à Claude Simon, Paris, Fayard.

- (2018) : «La langue littéraire à l'épreuve du temps », Acta fabula, vol. 19, n 1, « Dix ans de théorie », http://www.fabula.org/lodel/acta/document10657.php.

Quéré, Louis (2013) : « Les formes de l'événement », in E. Ballardini, R. Pederzoli, S. Reboul-Touré, G. Tréguer-Felten dirs, Les facettes de l'événement : des formes aux signes, mediAzioni 15, http:// mediazioni.sitlec.unibo.it

Rabatel, Alain et Petitjean, André dirs (2007) : Pratiques, 135-136, Questions de style.

Rastier, François (2001) : Arts et sciences du texte, Paris, PUF, coll. « Formes sémiotiques ».

Ricoeur, Paul (1992) : « Le retour de l'événement », Mélanges de l'École française de Rome, Italie et Méditerranée, vol.104, n¹, p. 29-35, www.persee.fr/doc/mefr_1123-9891_1992_num_104_1_4195

Rivalan Guégo Christiane, Rodrigues Denis dirs (2011) : L'Écho de l'événement. Du Moyen Âge à l'époque contemporaine, Presses universitaires de Rennes.

Romano, Claude (1998) : L'Événement et le monde, Paris, P.U.F., « Epiméthée ».

- (1999) : L’Événement et le temps, Paris, P.U.F., « Epiméthée ».

Sartenaer, Olivier (2018) : «Émergence », in M. Kristanek dir., L'Encyclopédie philosophique, en ligne, http://encyclo-philo.fr/emergence-a/

Sawyer, Robert Keith (2005) : Social Emergence. Societies as Complex Systems, Cambridge University Press.

Simiand, Paul (1903) : « Méthode historique et science sociale », Revue de synthèse historique, repris dans les Annales ESC, $1960, \mathrm{n}^{\circ} 1$, p. 83-119.

Tamm, Marek dir. (2015) : Afterlife of Events. Prespectives on Mnemohistory, Palgrave Macmillan.

Vallespir, Mathilde (2017) : «Énaction et pragmatique du discours : l'ironie comme processus discursif multistable », Signifiances (Signifying), 1 (1), p. 91-100.

Varela, Francisco, Thompson, Evan \& Rosch, Eleanor (1993) : L'Inscription corporelle de l'esprit. Sciences cognitives et expérience humaine, Paris, Seuil, coll. « La couleur des idées ».

Vinolo, Stéphane (2013) : « L'apostrophe de l'événement. Romano à la lumière de Badiou et Marion ", Journal of French and Francophone Philosophy - Revue de la Philosophie française et de langue française, XXI, 2, p. 51-67.

Watine, Marie-Albane (2017), « Norme psycholinguistique, norme esthétique : le cas de la tmèse ", in Lucile Gaudin-Bordes et Michèle Monte (dir.), Normes textuelles et discursives. Émergence, variations et conflits, Presses Universitaires de Franche-Comté, p. 75-93.

Watine, Marie-Albane et Yocaris, Ilias (2017) : «Le style comme événement. Appel à communication », https://www.fabula.org/actualites/le-style-comme-evenement_78926.php 
Yocaris, Ilias (2016a) : Style et semiosis littéraire, Paris, Classiques Garnier, coll. « Investigations stylistiques ».

(2016b) : « Les deux barricades : complexité sémiotique et objectivation des faits de style dans un extrait des Misérables », Semiotica, 213, p. 91-122.

\section{NOTES}

1. Une remarque orthographique s'impose: les Rectifications orthographiques $d u$ Conseil Supérieur de la Langue française, parues au BO du 6 décembre 1990, recommandent d'abandonner la graphie événement (qui prévalait depuis l'édition de $1740 \mathrm{du}$ Dictionnaire de l'Académie française, jusqu'à son édition de 1979) au profit de la graphie évènement, conforme à la prononciation et logiquement aligné sur avènement. Pour autant, les Rectifications en question "restent une référence mais ne sauraient être imposées » (BO 03/05/2012), et nous avons choisi de laisser les contributeurs libres d'utiliser l'une ou l'autre graphie.

2. Sur tous ces points, voir les synthèses de Fagot-Largeault 2002 et Sartener 2018.

\section{INDEX}

Mots-clés: style, événement, historicité, émergence, adresse

\section{AUTHOR}

\section{MARIE-ALBANE WATINE}

Université Nice Sophia Antipolis, UCA, CNRS, BCL, UMR 7320

Marie-Albane Watine est maître de conférences en stylistique à l'Université Côte d'Azur. Elle a notamment abordé la question de la vocalité chez Claude Simon (La voix et la frontière. Sur Claude Simon, Champion, 2007) ; elle travaille actuellement sur la notion de phrase en stylistique, appréhendée d'un point de vue psycholinguistique, historique ou figural (« Histoire de la phrase au XX ${ }^{\mathrm{e}}$ siècle », Gilles Siouffi dir., Histoire de la phrase française, Actes Sud, à paraître ; « Norme psycholinguistique, norme esthétique : le cas de la tmèse ", in Lucile Gaudin-Bordes et Michèle Monte (dir.), Normes textuelles et discursives. Émergence, variations et conflits, Presses Universitaires de Franche-Comté). 